\title{
Killip Class III
}

National Cancer Institute

\section{Source}

National Cancer Institute. Killip Class III. NCI Thesaurus. Code C77271.

A classification of myocardial infarction characterized by rales over more than $50 \%$ of the lung fields. 